\title{
Localization of genes causing postzygotic isolation in two hybridizations involving Drosophila pseudoobscura
}

\author{
H. Allen Orr
}

\author{
Department of Ecology and Evolution, \\ The University of Chicago, 1103 E. 57th St., \\ Chicago, Illinois 60637, U.S.A.
}

The genes causing postzygotic isolation in two Drosophila hybridizations were localized to regions of the $X$ chromosome. The $\mathrm{X}$ has a large effect on D. pseudoobscura pseudoobscura ("U.S.A.")-D. pseudoobscura bogotana ("Bogota") hybrid male sterility. Recombination analysis shows that this effect is due entirely to a small chromosome region representing less than 20 per cent of the $X$. This result suggests that a single $X$-linked gene may be involved. $A$ maximum likelihood mapping procedure places this hybrid sterility gene at position $50 \cdot 3(95$ per cent limits $=$ 46.9-54.4). Analysis also shows that this putative gene has a very large effect on hybrid fitness: substitution of a Bogota for a U.S.A. allele almost halves hybrid male fertility.

The X-chromosome also has a large effect on male and female sterility in the $D$. pseudoobscura-D.persimilis hybridization. Male sterility in this cross appears to involve more X-linked genes than in the younger U.S.A.-Bogota hybridization: genes on both arms of the $X$ are involved. Sterility of females, however, is caused by gene(s) restricted to the left arm of the $X$. Thus male and female sterility in this hybridization must involve some different loci. This result militates against the traditional explanation of Haldane's rule.

\section{INTRODUCTION}

There has been a resurgence of interest in the genetics of speciation. Recent workers have both determined the number of chromosome regions causing postzygotic isolation in Drosophila (see Coyne and Orr, 1989 $a$ for review), and have mapped the location of several genes causing hybrid sterility (Coyne and Charlesworth, 1986, 1989; Pantazidis and Zouros, 1988). These studies have several purposes: first, to estimate the number of genes causing reproductive isolation, and second, to determine whether different chromosome regions, and thus different genes, cause male and female postzygotic isolation (see Orr and Coyne, 1989). Third, precise mapping may facilitate molecular isolation and characterization of these "speciation genes". Finally, by analyzing several related hybridizations, one can determine whether certain regions or genes are especially likely to cause postzygotic isolation. Because we already know that the $\mathrm{X}$ chromosome plays a large role in hybrid sterility and inviability in Drosophila (Charlesworth et al., 1987), one would like to know whether these large $\mathrm{X}$-effects result from the action of the same regions in several hybridizations.
To address these questions, I localized the $\mathrm{X}$ linked genes causing hybrid sterility in two Drosophila hybridizations: D. pseudoobscura pseudoobscura (hereafter called "U.S.A.")-D. pseudoobscura bogotana (hereafter "Bogota") and D. pseudoobscura-D. persimilis. When Bogota females are crossed to U.S.A. males, sterile $F_{1}$ males and fertile females are produced. Males and females from the reciprocal cross are fertile (Prakash, 1972). The $\mathrm{X}$ has the largest effect of any chromosome on hybrid male fertility (Orr, 1989). Moreover, recombination analysis reveals that this large effect is due to a gene or genes on the left arm of the X near the yellow locus (I: $59 \cdot 3$ ). In fact, the $\mathrm{X}$-effect is due entirely to a region representing less than 30 per cent of the $\mathrm{X}$ chromosome (Orr, 1989). Here, I determine whether this effect is due to an even smaller region of the $X$. Assuming that this effect is due to a single locus, I also report the map position of this speciation gene.

The D. pseudoobscura-D. persimilis hybridization has been subject to extensive genetic analysis, beginning with Lancefield's (1929) and Dobzhansky's (1936) classic studies. These sibling species show substantial premating isolation (Lancefield, 
1929). Moreover, hybrid males produced in both reciprocal crosses are sterile (Lancefield, 1929). The $\mathrm{X}$ chromosome has the largest effect of any chromosome on hybrid male fertility (Orr, 1987).

Hybrid $F_{1}$ and backcross females are frequently semisterile, the severity depending on the strains employed (Lancefield, 1929; Orr, 1987). (As pointed out in the Discussion, "sterile" females actually produce zygotes which die before reaching the larval stage; as offspring lethality depends upon the mother's genotype [a maternal effect], this phenonemon is usually referred to as "female sterility" (Gottschewski, 1940; Mampell, 1941; Orr, 1987)). The $X$ chromosome has the largest effect on sterility among hybrid females produced in backcrosses to D. pseudoobscura (Mampell, 1941; Orr, 1987). Here I determine whether $D$. pseudoobscura- $D$. persimilis male and female sterility result from genes in one or both arms of the $X$ chromosome, and whether male and female sterility involve the same chromosome arms and thus possibly the same loci.

\section{METHODS AND MATERIALS}

Crosses were made between a U.S.A. strain carrying a multiply-marked $\mathrm{X}$ chromosome and a wildtype strain of either Bogota or $D$. persimilis. The hybrid $F_{1}$ females were backcrossed to the U.S.A. marker stock, yielding males and females carrying parental-type or recombinant X chromosomes. By assessing the fertility of these different genotypes, one can determine which regions of the X chromosome cause hybrid sterility.

In the U.S.A.-Bogota crosses, two U.S.A. stocks were used: one carrying Pointed (Pt I-0.0) yellow ( $y$ I-59.3) and the other carrying cut (ct I-21.9) yellow (all map locations from Anderson and Norman (1977)). Ideally, one would prefer to have all three markers present in the same stock but this was impossible as $P t$ and $c t$ are both wing shape mutations which cannot be reliably scored simultaneously (these are the only morphological markers available in this region of the $\mathrm{X}$ chromosome).

The $D$. pseudoobscura-D. persimilis crosses employed a yellow short (sh I-201.9) D. pseudoobscura stock. Yellow resides on the left arm of the $\mathrm{X}$ chromosome (XL), while short is on the right arm (XR). Unfortunately, the D. pseudoobscura and D. persimilis Standard (ST) X chromosome arrangements differ by two large inversions, one on each arm; these differences prevent recovery of most $\mathrm{X}$ chromosome recombinants between the species (Dobzhansky, 1936). To facilitate recovery of recombinants, I used the $D$. persimilis Sex Ratio (SR) stock, which is homosequential with the right arm of the $D$. pseudoobscura ST arrangement (Sturtevant and Dobzhansky, 1936). SR males produce mostly Xbearing sperm, and therefore produce daughters but almost no sons (Sturtevant and Dobzhansky, 1936). I crossed $D$. persimilis SR females to $D$. pseudoobscura $y$ sh males; the resulting $F_{1}$ females were then backcrossed to $D$. pseudoobscura $y$ sh males. Because short is very near the right end of the large $D$. pseudoobscura $\mathrm{X}$, yellow and short assort nearly independently in hybrid $F_{1}$ females.

Male fertility was tested by Coyne's (1984) method. A male was considered fertile if he had any motile sperm; a male with no motile sperm was considered sterile (all males were four days old). The fertility of a genotype was calculated as the percentage of males possessing any motile sperm. Female fertility was tested by Orr's (1987) method: a female who produced one or more larvae was considered fertile. If a female produced no larvae, her reproductive tract was removed in insect Ringer's solution and checked for the presence of sperm. If no sperm were found she was considered unmated. If sperm were present she was scored as sterile. Female fertility was calculated as the percentage of mated females who produced larvae.

All crosses were kept at $18^{\circ} \mathrm{C}$ for two days and then transferred to fresh vials and kept at $22^{\circ} \mathrm{C}$.

\section{RESULTS}

\section{Bogota-U.S.A. hybridization}

As expected, males from the pure species stocks are highly fertile (table 1 ). $F_{1}$ males with Bogota mothers, however, usually lack motile sperm (table 1); as noted by Prakash (1972) and Orr (1989), those rare hybrid males possessing motile sperm have extremely short sperm. All $\mathrm{F}_{1}$ males are sterile when tested with virgin females (Prakash, 1972; Orr, 1989).

Orr (1989) showed that recombination to the right of the yellow locus does not affect hybrid male fertility, implying that the gene(s) causing hybrid male sterility is (are) to the left of yellow. We must determine (a) whether recombination to the left of yellow affects male fertility, proving the existence of hybrid sterility gene(s) in this region, (b) whether these genes are between $P t(0 \cdot 0)$ and ct $(21.9)$, or between $c t$ and $y(59.3)$, or are found 
Table 1 Fertility of males and females from pure species stocks and from $F_{1}$ hybridizations. Abbreviations used: Bogota $=$ D. pseudoobscura bogotana; U.S.A. = D. pseudoobscura pseudoobscura; per $=D$. persimilis

\begin{tabular}{|c|c|c|}
\hline Males & Per cent fertile & $N$ \\
\hline Bogota-ER & $100 \cdot 0$ & 135 \\
\hline Pty (U.S.A.) & $99 \cdot 2$ & 127 \\
\hline ct y (U.S.A.) & $100 \cdot 0$ & 115 \\
\hline$y \operatorname{sh}$ (U.S.A.) & $97 \cdot 5$ & 81 \\
\hline $\mathrm{F}_{1} \delta(\mathrm{B} \circ \mathrm{g} q \times c t y \delta)$ & $2 \cdot 4$ & 209 \\
\hline $\mathrm{F}_{1} \delta($ per $\mathrm{SR} q \times y$ sh $\delta)$ & $0 \cdot 0$ & 207 \\
\hline Females & Per cent fertile & $N$ \\
\hline per SR & $100 \cdot 0$ & 78 \\
\hline$y \operatorname{sh}$ (U.S.A.) & $97 \cdot 0$ & 66 \\
\hline $\mathrm{F}_{1} q(y \operatorname{sh} q \times \operatorname{per} \mathrm{SR} \delta)$ & $98 \cdot 0$ & 151 \\
\hline
\end{tabular}

in both regions, and (c) the map position(s) of the gene(s) involved.

Experiment 1 of table 2 shows that recombination between $P t$ and $y$ affects hybrid male fertility. Thus, as expected, there are gene(s) to the left of $y$ causing hybrid sterility. There are two additional points to note. First, males carrying the U.S.A. $P t$ $y$ markers are highly fertile, despite the fact that most of them must carry some Bogota X chromosome material to the right of $y$ (approximately 70 per cent of the $\mathrm{X}$ chromosome is to the right of $y$ ). This again demonstrates that there are no genes to the right of $y$ with discernible effects on hybrid fertility.

Second, substitution of the Bogota $y^{+}$allele reduces fertility much more than substitution of the Bogota $\mathrm{Pt}^{+}$allele. While this suggests that the Bogota gene(s) causing sterility is (are) closer to $y$ than to $P t$, we cannot rule out the possibility that there are many genes between $P t$ and $y$ affecting hybrid fertility, but that those with the larger effects lie closer to $y$ than to $P t$.

To distinguish between these possibilities, I performed the ct $y$ backcross analysis (table 2, experiment 2). These data show that $c t y$ backcross males are as fertile as the Pt $y$ males of experiment $1(96.2$ vs. $95 \cdot 1$ per cent fertile, respectively; chisquare $=0.325, \mathrm{P}>0.55)$. This fact alone demonstrates that there are no genes causing hybrid sterility between Pt and ct: males with uncontrolled recombination between $P t$ and $c t$ (the $c t y$ males of experiment 2) are just as fertile as those males carrying little or no Bogota material in this region (the Pt $y$ males of experiment 1). Thus the X-linked gene(s) causing male sterility in the U.S.A.-Bogota hybridization must lie between $c t$ and $y$, a region
Table 2 Fertility of Bogota-U.S.A. hybrid males carrying recombinant and parental-type $X$ chromosomes. The males in Experiment 1 resulted from the cross $F_{1} q$ (Bogota $q \times$ Pt $y \quad$ o $) \times$ Pt $y \quad$. Males in Experiment 2 resulted from the cross $F_{1} q\left(\right.$ Bogota $q \times$ ct $\left.y \delta^{*}\right) \times$ ct $y \delta^{*}$

\begin{tabular}{|c|c|c|c|c|}
\hline Experimen & & & & \\
\hline Genotype & Pty & $P t^{+} y$ & Pt $y^{+}$ & $\mathrm{Pt}^{+} \mathrm{y}^{+}$ \\
\hline Fertile & $\begin{array}{l}95 \cdot 1 \% \\
N=223\end{array}$ & $\begin{array}{l}84 \cdot 5 \% \\
N=252\end{array}$ & $\begin{array}{l}63 \cdot 0 \% \\
N=351\end{array}$ & $\begin{array}{l}51 \cdot 4 \% \\
N=294\end{array}$ \\
\hline
\end{tabular}

Experiment 2

\begin{tabular}{lllll}
\hline Genotype & $c t y$ & $c t^{+} y$ & $c t y^{+}$ & $c t^{+} y^{+}$ \\
Fertile & $96 \cdot 2 \%$, & $88 \cdot 1 \%$, & $67 \cdot 4 \%$, & $57 \cdot 1 \%$, \\
& $N=210$ & $N=227$ & $N=141$ & $N=273$ \\
\hline
\end{tabular}

which represents less than 20 per cent of the $D$. pseudoobscura X chromosome.

While not proving it, the restriction of this effect to such a small region of the $\mathrm{X}$ strongly suggests that the large effect of the $\mathrm{X}$ is attributable to a single gene. Making this assumption, one can roughly estimate this gene's location from the data of experiment 2. On average, substitutions at the $y$ locus affect fertility $3 \cdot 28$ times more than substitutions at the $c t$ locus: substitution of the Bogota $y^{+}$allele into a $c t y$ genotype reduces fertility $3 \cdot 56$ times more than substitution of $\mathrm{Ct}^{+}$; conversely, substitution of the U.S.A. $y$ allele into a $c t^{+} y^{+}$ genotype increases fertility 3.01 times more than substitution of $c t$ (the small difference between the numbers 3.56 and 3.01 (average $=3.28$ ) shows that the substitutions are not exactly additive, i.e., $c t^{+}$ $y^{+}$males are slightly more sterile than expected from the individual effects of $c t^{+}$and $y^{+}$). The gene causing hybrid sterility is therefore about 3.28 times closer to $y$ than to $c t$. This places the hybrid sterility gene at approximately $50.6 \mathrm{cM}$, assuming that the map positions of $c t$ and $y$ are accurate.

This calculation, however, naively ignores the complications of double cross-overs. Also, it does not allow one to place confidence limits around the map position nor to estimate the average fertilities of the U.S.A. vs. Bogota alleles on the hybrid genetic background. These shortcomings are overcome by employing a maximum likelihood mapping procedure, similar to that advocated by Lander and Botstein (1989) (the present situation is greatly simplified because we are concerned only with one interval between two markers). For any given distance, $d$, separating the putative sterility gene and the yellow locus, one can find (by iteration) the fertilities of the U.S.A. allele $\left(f_{\text {U.S.A. }}\right)$ and the Bogota allele $\left(f_{\mathrm{BOG}}\right)$ at the sterility locus that maximize the likelihood of obtaining the 
observed data. One can then iteratively find the $\left(d, f_{\text {U.S.A. }}, f_{\mathrm{BOG}}\right)$ combination that provides the best overall fit to the observed fertility data (observed and expected data for any given $\left[d, f_{\text {U.S.A }}, f_{\mathrm{BOG}}\right]$ set are compared by G tests). A BASIC program, which assumes the Haldane mapping function, $r=(1-\exp (-2 d)) / 2$ (Lander and Botstein, 1989), was written to perform these iterations. Given the uncertainty surrounding the appropriate mapping function for $D$. pseudoobscura, only the Experiment 2 data were used, as the markers employed there are much closer to the putative sterility gene than in Experiment 1; indeed the Pointed locus of Experiment 1 is so far from the sterility gene that the two loci probably freely recombine (see position below).

The maximum likelihood procedure places the hybrid sterility gene at position 50.3 (95 per cent limits $=46 \cdot 9-54 \cdot 4)$, i.e., about $9 \cdot 0$ map units from the yellow locus. This position is very close to that estimated above by the simpler method. On the largely U.S.A. genetic background, males carrying the U.S.A. allele have an estimated fertility of 98 per cent while males carrying the Bogota allele have a fertility of only 57 per cent. Thus substitution of a Bogota allele at this putative locus has a very large effect on hybrid fitness: male fertility is almost halved. The fertility data expected given a single gene with $\left(d, f_{\text {U.S.A. }}, f_{\mathrm{BOG}}\right)=(9 \cdot 0,0 \cdot 98,0 \cdot 57)$ fit the observed data very well $\left(\mathrm{G}_{1}=0 \cdot 215, P>\right.$ $0 \cdot 60$ ). The results in table 2 are thus quite consistent with a single-locus interpretation.

\section{D. pseudoobscura-D. persimilis hybridization}

Males and females from the pure species stocks are highly fertile (table 1 ). Hybrid $F_{1}$ males are completely sterile, as expected, while hybrid $F_{1}$ females are highly fertile (table 1). Hybrid males and females, however, are frequently sterile in the backcross generation (table 3 ).

The $\mathrm{X}$ chromosome obviously has a large effect on male fertility: $y$ sh males, who carry a $D$. pseudoobscura X chromosome on a predominantly D. pseudoobscura genetic background, are often fertile, while $y^{+} s h^{+}$males, who carry a $D$. persimilis X chromosome, are always sterile (table 3 ).

Use of the $D$. persimilis SR stock permitted recovery of a large number of $X$ chromosome recombinants, allowing one to assess the effect of each chromosome arm on hybrid fertility. Substitution of the $D$. persimilis $y^{+}$for the $y$ allele has a very large and significant effect on male fertility (chi-square $=76 \cdot 07, P<0 \cdot 0001$ ). Substitution of
Table 3 Fertility of $D$. pseudoobscura- $D$. persimilis hybrid backcross males and females. Hybrids produced from $F_{1} \&$ (per SR/SR $q \times y$ sh $\delta) \times y$ sh $\delta$ cross

\begin{tabular}{lll}
\hline Males & Per cent fertile & $N$ \\
\hline$y s h$ & $22 \cdot 1$ & 154 \\
$y s h^{+}$ & $11 \cdot 1$ & 126 \\
$y^{+} s h$ & $0 \cdot 0$ & 229 \\
$y^{+} s h^{+}$ & $0 \cdot 0$ & 184 \\
\hline Females & Per cent fertile & $N$ \\
\hline$y s h$ & $75 \cdot 6$ & 41 \\
$y s h^{+}$ & $82 \cdot 0$ & 61 \\
$y^{+} s h$ & $47 \cdot 9$ & 280 \\
$y^{+} s h^{+}$ & $41 \cdot 6$ & 315 \\
\hline
\end{tabular}

the $D$. persimilis $s h^{+}$allele has a smaller but still significant effect on fertility (chi-square $=$ $5 \cdot 87, P<0 \cdot 02$ ). (Unfortunately, a multivariate analysis of the effects of these substitutions over all genetic backgrounds is uninformative because substitution of the $D$. persimilis $y^{+}$allele causes complete sterility; one cannot, therefore, determine whether substitution of the $s h^{\dagger}$ allele further decreases fertility on a $y^{+}$background or whether there are any interactions between the two substitutions. One must therefore use univariate statistics.) Thus, as Wu and Beckenbach (1983) showed previously, genes on both arms of the $D$. persimilis $\mathrm{X}$ chromosome cause male sterility on a predominantly $D$. pseudoobscura genetic background. We can now compare these results with those obtained for females, which have not been previously investigated.

Females carrying a $D$. pseudoobscura y sh X chromosome on a largely $D$. pseudoobscura genetic background are usually fertile ( 75.6 per cent, table 3 ). Substitution of the region of the $D$. persimilis $\mathrm{X}$ chromosome linked to the $y^{+}$allele, however, significantly decreases fertility (table 3 , maximum likelihood chi-square $=34 \cdot 86, P<0 \cdot 0001 ;$ all statistics from multivariate CATMOD procedure from SAS, Inc.). Substitution of the $D$. persimilis $s h^{+}$allele does not however significantly affect female fertility $($ maximum likelihood chi-square $=$ $0 \cdot 06, P=0 \cdot 80)$. There is also no significant interaction of the chromosome regions on fertility ( maximum likelihood chi-square $=1 \cdot 50, P=0 \cdot 22$ ).

Thus, unlike the genes affecting males, the $\mathrm{X}$-linked genes causing hybrid female sterility are apparently limited to the left arm of the X. $D$. pseudoobscura-D. perimilis hybrid male and female sterility must therefore involve some different X-linked genes. 


\section{DISCUSSION}

There has been a great deal of speculation about the number of genes involved in speciation: Dobzhansky (1937) emphasized that reproductive isolation in animals evolved gradually and so is probably highly polygenic. Goldschmidt (1940) and, more recently, Stanley (1979) have suggested that speciation in animals occurs very rapidly and involves one or a very few genes of large effect.

Recent genetic analyses of postzygotic isolation in Drosophila provide support for an intermediate position. As Orr and Coyne (1989) note, particular forms of isolation (e.g., non-reciprocal hybrid male sterility) sometimes appear to have a simple genetic basis: a few chromosome regions have a large effect on postzygotic isolation, while other large segments have no discernible effect. These results suggest that a fairly small number of loci are involved.

Two lines of evidence, however, demonstrate that reproductive isolation evolves gradually and so involves an increasing number of loci through time. First, comparison of the strength of reproductive isolation with the age of taxa pairs (as estimated by electrophoretic genetic distance) shows that reproductive isolation increases with time; at least in allopatry, there is no sudden jump from little or no to strong reproductive isolation (Coyne and Orr, 1989b). Second, when one compares the genetic basis of isolation between taxa showing weak vs. strong reproductive isolation, more loci are apparently involved in the latter than in the former (Orr, 1989; Orr and Coyne, 1989). This need not, of course, be the case: it is entirely possible a priori that weakly isolated taxa have simply diverged at a few loci with small effects while strongly isolated taxa have fortuitously diverged at an equally small number of genes but with each gene having a fairly large effect. Thus, while any particular form of reproductive isolation may involve a small number of genes, the number of genes responsible for "species-level" reproductive isolation could be fairly large.

The present work supports this view. The $D$. pseudoobscura Bogota-USA hybridization is fairly young (Nei's $D=0 \cdot 194$; Ayala and Dobzhansky, 1974) and shows weak postzygotic isolation (nonreciprocal male sterility) and no prezygotic isolation (Prakash, 1972). While the X chromosome has a large effect on hybrid male sterility (Orr, 1989), the data reported here show that this effect is entirely attributable to factor(s) between the cut and yellow loci on the left arm of the $\mathrm{X}$, a region representing less than 20 per cent of the $X$. This result strongly suggests the involvement of a single gene (the probability of two randomly placed Xlinked genes occurring in this region is less than $0 \cdot 20^{2}=0 \cdot 04$ ). Assuming the involvement of a single gene, maximum likelihood analysis places this locus at position 50.3 (95 per cent limits = 46.9-54.4). This putative gene has a very large effect on hybrid fitness: substitution of a Bogota for a U.S.A. allele approximately halves male fertility. Analysis of the region near the yellow locus using restriction fragment markers could, of course, allow more precise mapping of this gene; molecular characterization of this speciation gene might then be possible.

D. pseudoobscura-D. persimilis (Nei's $D=$ $0 \cdot 30$; Ayala and Dobzhansky, 1974) is older than the D. pseudoodbscura Bogota-U.S.A. hybridization. Not surprisingly, D. pseudoobscura and $D$. persimilis also show stronger reproductive isolation (see Introduction). The present analysis shows that hybrid male sterility is caused by genes on both arms of the X, confirming Wu and Beckenbach's (1983) results: $D$. persimilis genes linked to the yellow locus on XL and to the short locus on XR reduce hybrid male fertility when on a predominantly $D$. pseudoobscura genetic background. Thus, male sterility in this older hybridization appears to involve more $\mathrm{X}$-linked genes than in the Bogota-U.S.A. cross. Indeed, combining the results of Lancefield's (1929) and Wu and Beckenbach's (1983) studies shows that D. pseudoobscura-D. persimilis male sterility involves at least 4 or $5 \mathrm{X}$-linked loci.

D. pseudoobscura-D. persimilis hybrid females are also frequently sterile (Lancefield, 1929; Mampell, 1941; Orr, 1987). The X chromosome has the largest effect on the fertility of hybrid females produced in backcrosses to D. pseudoobscura: females carrying a $D$. persimilis $\mathrm{X}$ chromosome on a predominantly $D$. pseudoobscura genetic background often fail to produce offspring (Mampell, 1941; Orr, 1987). The present study shows that this effect is due to gene(s) on the left, but not right, arm of the $D$. persimilis $X$ chromosome (at least, there are no genes affecting female fertility linked to the short locus on XR).

Because the short region of the $\mathrm{X}$ chromosome affects hybrid male but not female sterility, $D$. pseudoobscura-D. persimilis male and female sterility must involve some different loci. This finding is perhaps not surprising given what is known about the different modes of action of these genes: male sterility results from genes that act within the hybrid males themselves (Orr, 1987), while female "sterility" actually results from the death of a 
female's offspring due to her maternally-acting genes (Gottschewski, 1940; Mampell, 1941). However, because genes are often both maternallyand zygotically-acting (Underwood and Lengyel, 1988), this distinction alone does not prove that different genes cause male and female sterility. Interestingly, in the only previous comparison of the genetics of male and female postzygotic isolation, Orr and Coyne (1989) found that D. virilis - D. novamexicana hybrid male and female sterility also result from the action of different X-linked genes.

The finding that male and female postzygotic isolation result from different genes militates against the traditional explanation of Haldane's rule (the preferential sterility or inviability of hybrids of the heterogametic sex; in Drosophila, males). Dobzhansky (1937) argued that heterogametic hybrids suffer from an X-autosomal imbalance: while homogametic hybrids carry one $\mathrm{X}$ and one haploid set of autosomes from each species, heterogametic hybrids lack an $\mathrm{X}$ that is compatible with one set of autosomes. Muller $(1940,1942)$ further suggested that most substitutions on the $X$ are recessive; these genes will obviously affect hetero- far more than homogametic hybrids. Thus, according to Muller, the genes causing male sterility/inviability would also affect females if the substituted alleles were partially dominant (conversely, those rare dominant genes that affect hybrid females would also affect males).

The finding that different sets of genes cause male and female hybrid sterility/inviability suggests, however, that Haldane's rule simply results from the more rapid accumulation of genes affecting male than female hybrids. Coyne and Orr (1989a) have offered a population genetic theory to explain this differential rate of substitution in the two sexes. Orr and Coyne (1989) also discuss several other lines of evidence falsifying Muller's explanation of Haldane's rule. Analysis of other hybridizations is obviously needed to determine whether male and female postzygotic isolation usually result from the action of different genes.

Last, it is interesting that the left arm of the $X$ chromosome has the largest effect in all three cases of hybrid sterility analyzed here. In the case of $D$. pseudoobscura-D. persimilis male sterility this may, of course, merely reflect lower recombination about the XL than the XR marker in $F_{1}$ females (i.e., D. pseudoobscura is homosequential with the right, but not the left, arm of the $D$. persimilis Sex Ratio X chromosome). This is not a likely explanation of the $D$. pseudoobscura-D. persimilis female sterility data, however, as XR has no significant effect whatever. Also, this explanation cannot account for the larger effect of XL in the $D$. pseudoobscura Bogota-U.S.A. hybridization as these taxa are homosequential for the entire X. Orr and Coyne (1989) similarly found that the same region of the $\mathrm{X}$ chromosome (near the $D$. virilis white locus) causes sterility in several evolutionarily independent hybridizations in the virilis group; indirect evidence showed that other regions of the $X$ have no discernible effect on fertility. Thus we cannot yet rule out the possibility that certain chromosome regions, and thus genes, are especially likely to cause postzygotic isolation. Analysis of other sets of closely related species is necessary to determine if this pattern is real or spurious.

Acknowledgements I thank J. Coyne for his valuable comments on this manuscript. I also thank an anonymous reviewer for suggesting and outlining the mapping procedure used. This work was supported by training grant GM 07197 from the National Institute of General and Medical Sciences of the National Institutes of Health to the University of Chicago, and by National Institutes of Health grant GM 38462 to J. Coyne.

\section{REFERENCES}

ANDERSON, W. AND NORMAN, R. 1977. Brief descriptions and map positions of currently-available mutants of $D$. pseudoobscura. Drosophila Inform. Serv., 52, 11-12.

AYALA, F. J. AND DOBZHANSKY. T. 1974. A new subspecies of Drosophila pseudoobscura (Diptera: Drosophilidae). Pan-Pacific Entomol., 50, 211-219.

CHARLESWORTH, B., COYNE, J. AND BARTON, N. 1987. The relative rates of evolution of sex chromosomes and autosomes. Am. Nat., 130, 113-146.

COYNE, J. A. 1984. Genetic basis of male sterility in hybrids between two closely related species of Drosophila. Proc. Natl Acad. Sci. USA, 81, 4444-4447.

COYNE, J. AND CHARLESWORTH, B. 1986. Location of an $\mathrm{X}$-linked factor causing sterility in male hybrids of Drosophila simulans and D. mauritiana. Heredity, 57, 243246.

COYNE. J. AND CHARLESWORTH, B. 1989. Genetic analysis of $\mathrm{X}$-linked sterility genes in hybrids between three sibling species of Drosophila. Heredity, 62, 97-106.

COYNE, J. AND ORR, H. 1989a. Two rules of speciation. In Otte, D. and Endler, J. (eds), Speciation and Its Consequences, Sinauer Associates, Sunderland, Mass.

COYNE, J. AND ORR, H. 1989 b. Patterns of speciation in Drosophila. Evolution, 43, 362-381.

DOBZHANSKY, T. 1936. Studies on hybrid sterility. II. Localization of sterility factors in Drosophila pseudoobscura hybrids. Genetics, 21, 113-135.

DOBZHANSKY, T. 1937. Genetics and the Origin of Species. Columbia University Press, New York.

GOLDSCHMIDT, R. 1940. The Material Basis of Evolution. Yale University Press, New Haven, Conn.

GOTTSCHEWSKI, G. 1940. Eine analyse bestimmter Drosophila pseudoobscura- Rassen- und artkreuzungen. Z. Indukt. Abstam. Vererbung, 78, 338-398. 
LANCEFIELD, D. E. 1929. A genetic study of crosses of two races or physiological species of Drosophila obscura. $Z$. Indukt. Abstam. Vererbung, 52, 287-317.

LANDER, E. S. AND BOTSTEIN, D. 1989. Mapping Mendelian factors underlying quantitative traits using RFLP linkage maps. Genetics, 121, 185-199.

MAMPELL, K. 1941. Female sterility in interracial hybrids of Drosophila pseudoobscura. Proc. Natl Acad. Sci. USA, 27, 337-341.

MULLER, H. J. 1940. Bearing of the Drosophila work on systematics. pp. 185-268. In: Huxley, J. S. (ed.) The New Systematics, Clarendon Press, Oxford.

MULLER, H. J. 1942. Isolating mechanisms, evolution, and temperature. Biol. Symp., 6, 71-125.

ORR, H. A. 1987. Genetics of male and female sterility in hybrids of Drosophila pseudoobscura and D. persimilis. Genetics, 116, 555-563.

ORR, H. A. 1989. Genetics of sterility in hybrids between two subspecies of Drosophila. Evolution, 43, 180-189.

ORR, H. AND COYNE, J. 1989. The genetics of postzygotic isolation in the Drosophila virilis group. Genetics, 121, 527-537.
PANTAZIDIS, A. C. AND ZOUROS, E. 1988. Location of an autosomal factor causing sterility in Drosophila mojavensis males carrying the Drosophila arizonensis Y chromosome. Heredity, 60, 299-304.

PRAKASH, S. 1972. Origin of reproductive isolation in the absence of apparent genic differentiation in a geographic isolate of Drosophila pseudoobscura. Genetics, 72, 143-155.

StANley, S. M. 1979. Macroevolution: Pattern and Process. Freeman, San Francisco.

STURTEVANT, A. AND DOBZHANSKY, T. 1936. Geographical distribution and cytology of sex-ratio in Drosophila pseudoobscura and related species. Genetics, 21, 473-490.

WU, C.-I., AND BECKENBACH, A. 1983. Evidence for extensive genetic differentiation between the sex-ratio and the standard arrangement of Drosophila pseudoobscura and $D$. persimilis and identification of hybrid sterility factors. Genetics, 105, 71-86.

UNDERWOOD, E. AND LENGYEL, J. 1988. Drosophila genes encoding maternal-specific and maternal-differential RNAs. Devel. Genetics, 9, 23-35. 